\title{
Octadecadienoic Acid n-4
}

National Cancer Institute

\section{Source}

National Cancer Institute. Octadecadienoic Acid n-4. NCI Thesaurus. Code C68400.

A polyunsaturated long-chain fatty acid with an 18-carbon backbone and double bonds originating from the 4 th position and either the 7 th position or the 8 th position from the methyl end. There are at least 4 different stereoisomers that can be called by this name. 